\title{
Anti-epileptic prescribing patterns in the South African private health sector (2008-2013)
}

\author{
Karen Jacobsa , Marlene Julyan ${ }^{\text {iD }}$, Martie S Lubbe ${ }^{a}$, Johanita R Burgera* and Marike Cockeran ${ }^{a}$ iD \\ aMedicine Usage in South Africa, Faculty of Health Sciences, North-West University, Potchefstroom Campus, Potchefstroom, South Africa \\ *Corresponding author, Email: Johanita.Burger@nwu.ac.za
}

Abstract (Full text available online at www.tandfonline.com/ojfp)

S Afr Fam Pract 2016; DOI:10.1080/20786190.2016.1148337

Background: Little is known about longitudinal prescribing practices for anti-epileptic drugs (AEDs) in South Africa. The prescribing patterns and associated direct medicine costs of AEDs in the private health sector were investigated, using claims data from January 1, 2008 to December 31, 2013.

Methods: The annual prevalence of prescriptions, AEDs and AED generics per patient with epilepsy (ICD-10 code G40) was determined. Cost analyses conducted included the calculation of the total direct cost of AEDs (medical scheme contribution, patient co-payment, and single exit price (SEP)), and the average cost per AED per year.

Results: Prevalence of patients claiming anti-epileptics ranged between $0.87 \%$ and $0.91 \%$ from 2008 to 2013 . AED prescriptions/ patient ranged from $11.76(95 \% \mathrm{Cl}, 11.56-11.95)]$ in 2008 to $11.90(95 \% \mathrm{Cl}, 11.71-12.09)$ in 2013. Patients aged $40-65$ years had the highest number of AED prescriptions/year. Valproate was most prescribed, followed by lamotrigine and carbamazepine. Average cost per AED increased from R237.12 (95\% Cl, 233.58-240.65) in 2008 to R522.32 (95\% Cl, 515.24-529.41) in 2013, while the average patient co-payments increased from R27.76 (95\% Cl, 26.63-28.89) to R264.32 (95\% Cl, 260.61-268.03). Prescribing of generics increased by $12.84 \%$.

Conclusions: Generic prescribing increased over time; however, patient co-payments increased dramatically.

Keywords: anti-epileptic, direct medicine costs, longitudinal, medicine claims database, prescribing patterns, South Africa

\section{More than scales and tape measures needed to address obesity in South Africa}

\author{
R Ramlala and RD Govendera* \\ ${ }^{a}$ Department of Family Medicine, University of KwaZulu-Natal, Durban, South Africa \\ *Corresponding author, email: Govenderr1@ukzn.ac.za
}

Abstract (Full text available online at www.tandfonline.com/ojfp)

S Afr Fam Pract 2016; DOI:10.1080/20786190.2016.1151643

Obesity is an emerging public health problem worldwide increasing from 857 million in 1980 to 2.1 billion by 2013. A study was done on a sample of 100 obese and overweight patients with a body mass index (BMI) above $25 \mathrm{~kg} / \mathrm{m}^{2}$. The patients were asked to complete a questionnaire, which included socio-demographic data and perceptions regarding their weight.

The patients were between the ages of 18 and 76 years old with $82 \%$ being female patients. The mean BMI for males was $41.7 \mathrm{~kg} / \mathrm{m}^{2}(\mathrm{SD}=7.38112)$ and females $39.9 \mathrm{~kg} / \mathrm{m}^{2}(\mathrm{SD}=7.90504)$. The results of this study confirm that $17 \%$ of overweight and obese patients saw themselves as having a normal weight and $97 \%$ felt that they were not unattractive. Of the sample, $96 \%$ affirmed that being obese was a health risk. The commonest reason cited for their obesity is by choice (70\%).

Although obese patients knew that obesity is a health risk, they have a positive image of obesity. The dramatic trend towards increasing obesity suggest that healthcare providers need to understand how people from different cultures view obesity. This will help them to promote key messages about the health risks associated with excess weight in a culturally sensitive way.

Keywords: obesity, overweight, perceptions, South Africa 